Reporte de caso

\title{
Drenaje percutáneo trans-gástrico de necrosis pancreática amurallada complicado en paciente pediátrico
}

\section{WON transgastric percutaneous drainage complicated in a pediatric patient}

Carlos Maximiliano Priarone ${ }^{1 凶}$, María Virginia Pinzón-Fernández ${ }^{2 凶}$, Valentina Patiño-Pérez ${ }^{3 凶}$, Margarita María Benavides-Ruiz ${ }^{4 凶}$, Randol Karami Silva-Bermudez ${ }^{3 凶}$

${ }^{1}$ Médico, especialista en Cirugía General y Percutánea, profesor Universidad del Cauca, Popayán, Colombia.

${ }^{2}$ PhD, MPH. Profesora titular, Departamento de Medicina Interna, Universidad del Cauca, Popayán, Colombia.

${ }^{3}$ Estudiante de Medicina, Universidad del Cauca, Popayán, Colombia.

${ }^{4}$ Médico general, Pontificia Universidad Javeriana, Bogotá, Colombia.

Fecha correspondencia:

Recibido: febrero 02 de 2020.

Revisado: septiembre 29 de 2021.

Aceptado: octubre 04 de 2021.

\section{Forma de citar:}

Priarone CM, Pinzón-Fernández

MV, Patiño-Pérez V, Benavides-

Ruiz MM, Silva-Bermudez RK.

Drenaje percutáneo trans-gástrico

de necrosis pancreática

amurallada complicado en

paciente pediátrico. Rev CES Med

2021; 35(3): 316-324.

https://dx.doi.org/10.21615/

cesmedicina. 5443

Open access

(C) Derecho de autor

Licencia creative commons

Ética de publicaciones

Revisión por pares

Gestión por Open Journal System

\section{Resumen}

Las complicaciones de la pancreatitis aguda se dividen en sistémicas y locales, y entre estas últimas se encuentran las colecciones líquidas pancreáticas agudas, la colección necrótica aguda, el pseudoquiste y la necrosis amurallada pancreática. Se reporta el caso de niña de nueve años con pancreatitis aguda idiopática severa, quien desarrolló una necrosis pancreática amurallada de $75 \times 45 \mathrm{~mm}$. Por la persistencia del dolor abdominal y la intolerancia a la vía oral, a pesar del manejo médico, se indicó un abordaje percutáneo transgástrico para su tratamiento. Se obtuvo éxito técnico y terapéutico a través del drenaje de la colección, permitiendo iniciar la vía oral y disminuyendo los requerimientos de analgesia. La paciente evolucionó satisfactoriamente, con alta quirúrgica a los 13 días y retiro del catéter a los 30 días. A los cuatro meses de seguimiento se evidenció una colección recidivante asintomática, manejándose de manera conservadora. Lo anterior permite considerar que el abordaje percutáneo transgástrico es una técnica segura y eficaz; sin embargo, evaluar la efectividad y morbimortalidad en 
DOI:

10.21615/cesmedicina.5443

ISSNe 2215-9177

ISSN 0120-8705

Publica con nosotros población pediátrica requiere de otros estudios que posibiliten llegar conclusiones que generen mayor evidencia científica.

Palabras clave: pancreatitis aguda necrotizante; procedimientos quirúrgicos mínimamente invasivos; seudoquiste pancreático; fístula pancreática; páncreas.

\section{Abstract}

The complications of acute pancreatitis are divided into systemic and local such as acute pancreatic fluid collections, acute necrotic collection, pseudocyst and pancreatic walled necrosis. The case of a nine-year-old girl with idiopathic severe acute pancreatitis who developed a pancreatic walled necrosis of $75 \times 45 \mathrm{~mm}$ is reported. Due to the persistence of abdominal pain and intolerance to the oral route, despite medical treatment, a trans gastric percutaneous approach was indicated for the treatment of pancreatic walled necrosis. Technical and therapeutic success was obtained through the drainage of the collection, allowing to start the oral route and decreasing the analgesia requirements. The patient evolved satisfactorily, with surgical discharge after 13 days and removal of the catheter after 30 days. After 4 months of follow-up, a recurrent asymptomatic collection was evidenced, being handled in a conservative manner. The above mentioned allows considering that the trans-gastric percutaneous approach is a safe and effective technique; however, evaluating the effectiveness and morbimortality in pediatric population requires other studies that make possible to reach conclusions that generate more scientific evidence.

Keywords: acute necrotizing pancreatitis; minimally invasive surgical procedures; pancreatic pseudocyst; pancreatic fistula; wall off necrosis.

\section{Introducción}

La pancreatitis aguda es un proceso reversible con inflamación del parénquima pancreático, presencia de edema intersticial, grados variables de necrosis y, en ocasiones, hemorragia. Epidemiológicamente, se describe como una enfermedad frecuente con incidencia de 13,2 por cada 100000 niños ${ }^{(1)}$, aumentando en las últimas décadas ${ }^{(2)}$. En adultos, la etiología se relaciona con cálculos biliares y consumo de alcohol, mientras que en los niños se asocia a traumatismos, infecciones, anomalías congénitas y casos idiopáticos ${ }^{(3)}$. 
El síntoma más frecuente en los niños es el dolor (80-95\%) seguido por náuseas o vómito $(40-80 \%)^{(4,5)}$. Las complicaciones de la pancreatitis se clasifican en sistémicas, como el choque - la falla multiorgánica y, locales, como las colecciones líquidas pancreáticas agudas, la colección necrótica aguda, pseudoquiste y la formación de necrosis pancreática amurallada, conocida por sus siglas del inglés WON (Walled off Necrosis) ${ }^{(5,6)}$. En relación con lo anterior, el $20 \%$ de los casos de pancreatitis severas desarrollan necrosis pancreática, que transcurridas cuatro semanas se convierten en WON que suele infectarse en $30 \%$ de los casos ${ }^{(7)}$. En este sentido, la necrosis infectada está relacionada con tasas de morbi-mortalidad que oscilan entre el $8-39 \%{ }^{(7)}$.

Se reporta el caso de niña con diagnóstico de pancreatitis aguda severa idiopática quien desarrolló necrosis pancreática amurallada y su posterior tratamiento y evolución.

\section{Caso clínico}

Paciente femenina de nueve años quien ingresó al servicio de urgencias presentando un cuadro clínico de ocho horas de evolución caracterizado por dolor abdominal en hemiabdomen inferior, asociado a emesis, deposiciones diarreicas y alzas térmicas no cuantificadas. Al examen físico la paciente estaba hemodinámicamente estable, sin signos de dificultad respiratoria, presentaba dolor a la palpación en epigastrio y en ambos hipocondrios, sin signos de irritación peritoneal.

Se tomaron paraclínicos que evidenciaron leucocitos: $15100 / \mathrm{mm}^{3}$, con $82 \%$ de neutrófilos, hemoglobina $14 \mathrm{~g} / \mathrm{dL}, \mathrm{PCR} 12 \mathrm{mg} / \mathrm{L}$, amilasa $1368 \mathrm{U} / \mathrm{L}$ (valores de referencia: 20-96) y acidosis metabólica. Las pruebas de función renal y hepática fueron normales y el parcial de orina fue negativo para infección. La ecografía abdominal demostró dimensiones pancreáticas aumentadas, alteración difusa de su eco estructura y líquido laminar peri pancreático, por lo cual se hizo diagnóstico de pancreatitis aguda. Al séptimo día de hospitalización y debido a una tórpida evolución se trasladó a la unidad de cuidados intensivos y se realizó una tomografía contrastada de abdomen, la cual reportó páncreas aumentado de tamaño, necrosis de la cabeza y cuerpo del páncreas, colección peri pancreática en relación con su cola.

El cuadro clínico se clasificó como una pancreatitis aguda severa necrotizante, colección necrótica aguda con sospecha de infección, por lo cual se inició esquema antibiótico, se suspendió vía oral y se solicitó valoración por Nutrición y Cirugía que, al día 18 de 
hospitalización, solicitó una nueva tomografía que evidenció colección en cola de páncreas de aproximadamente $75 \mathrm{~mm} \times 45 \mathrm{~mm}$, con volumen subjetivo de $400 \mathrm{cc}$, la cual generaba compresión extrínseca del estómago (figura 1).

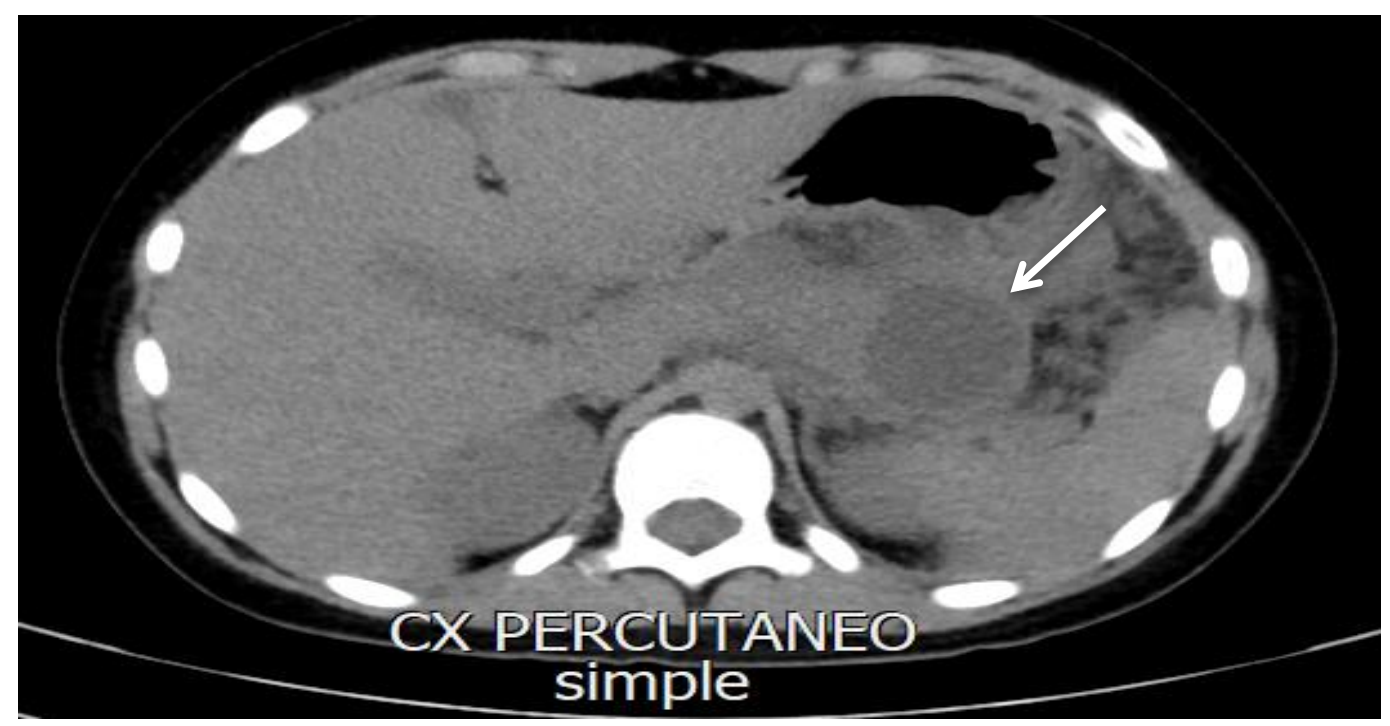

Figura 1. Colección en cola del páncreas en relación con la cara posterior del estómago.

En el día 24 de hospitalización presentó intolerancia a los alimentos sólidos, por lo cual se evaluó la posibilidad de un drenaje percutáneo de la colección. No se evidenció respuesta inflamatoria sistémica, por lo cual se decidió ajustar la analgesia y continuar con manejo expectante; sin embargo, el día 30 de hospitalización y dada la persistencia de dolor postprandial, se decidió realizar un drenaje percutáneo trans gástrico guiado por tomografía.

Intra-operatoriamente, se realizó tomografía contrastada de abdomen que evidenció necrosis pancreática amurallada (WON) relacionada con la cola del páncreas y cara posterior del estómago la cual medía $5 \times 5 \mathrm{~cm}$, aproximadamente. Se realizó punción del WON pancreático con aguja de 18G, atravesando el estómago (figuras $2 \mathrm{~A}$ y $2 \mathrm{~B}$ ). Se obtuvo líquido turbio necro hemorrágico que fue enviado para cultivo (con posterior resultado negativo) y dosificación de la amilasa cuyo valor fue de 2 504. Luego se procedió a la colocación de catéter transgástrico multipropósito de $8 \mathrm{fr}$ utilizando una guía teflonada, logrando drenar $40 \mathrm{ml}$ de líquido pancreático. Una tomografía de control verificó la correcta ubicación del catéter. En el postoperatorio inmediato se inició octeotride en infusión continua durante una semana. 

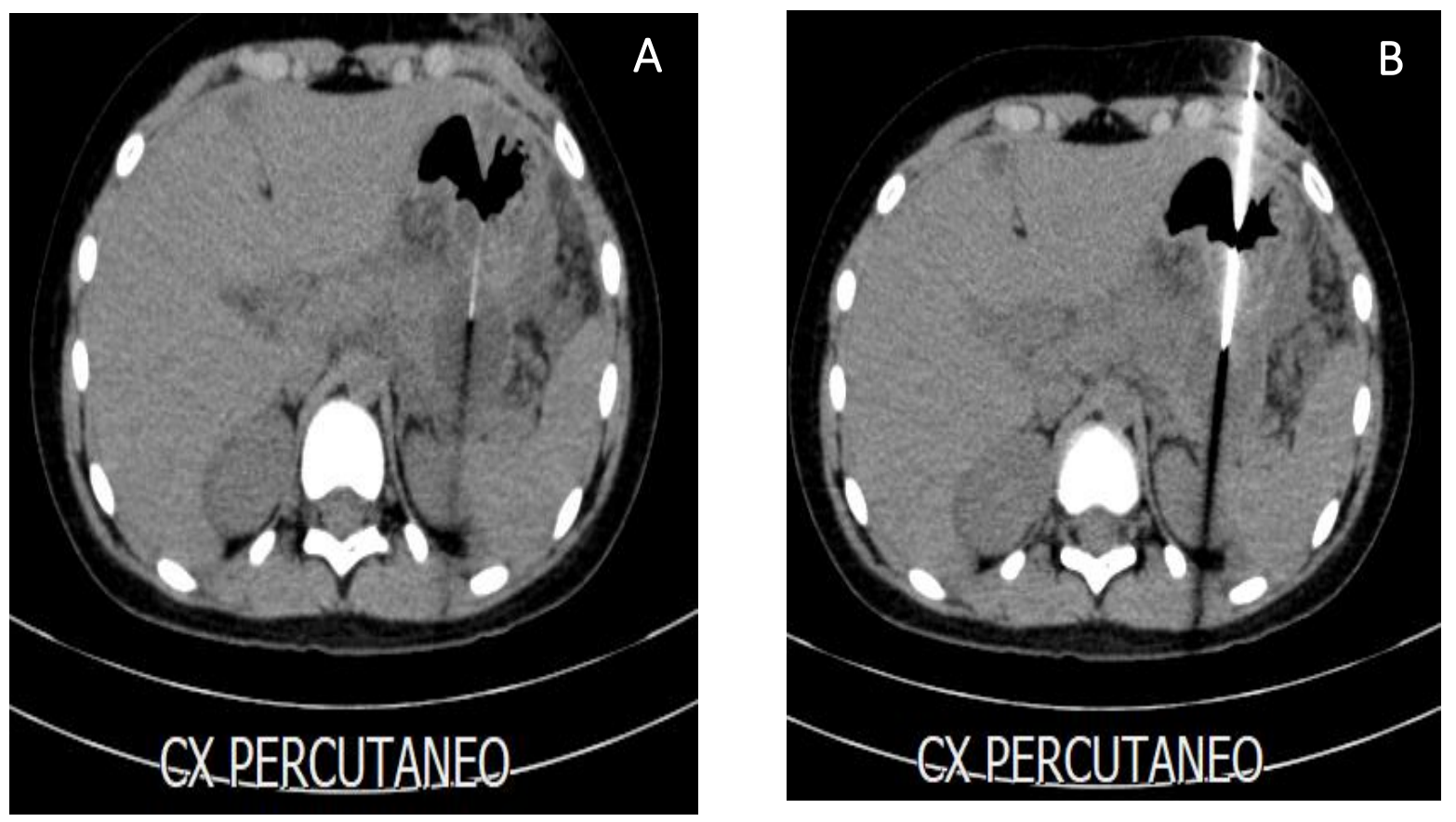

Figura 2. A y B. Punción transgástrica guiada por TAC.

Se evidencia la aguja de $18 \mathrm{G}$ atravesando las dos caras del estómago y ubicándose dentro de la colección.

La paciente evolucionó favorablemente después del drenaje percutáneo transvisceral, desapareciendo el dolor y tolerando adecuadamente la vía oral. Se indicó el egreso de la paciente sin retirar el catéter, debido a que persistía con escaso drenaje de líquido pancreático. Durante el seguimiento clínico e imagenológico se reportó buena evolución; no obstante, al mes y medio del posoperatorio, se evidenció desplazamiento del pigtail hacia la cavidad gástrica con ausencia de colecciones pancreáticas residuales, lo cual sucedió por tracción involuntaria del catéter y por esta razón se decidió retirarlo definitivamente. En un control ambulatorio a los cuatro meses posquirúrgicos se realizó una tomografía abdominal que evidenció una colección en cola de páncreas de $5 \times 3 \mathrm{~cm}$ (colección recidivante), la cual era asintomática y no requirió reintervención.

\section{Discusión}

La pancreatitis aguda desarrolla complicaciones locales que requieren abordajes mini-invasivos para su resolución; siendo una causa frecuente la idiopática ${ }^{(5,8,9)}$, coincidiendo con el caso descrito. El desarrollo de un WON es frecuente tras la presentación de una pancreatitis necrotizante severa ${ }^{(3)}$; no obstante, la literatura disponible sobre su tratamiento en niños es 
limitada. Si el manejo conservador fracasa, pueden utilizarse diferentes abordajes que incluyen drenaje endoscópico, drenaje percutáneo guiado por imágenes y necrosectomía abierta ${ }^{(10-12)}$. Esta última es utilizada cuando no se cuenta con procedimientos mínimamente invasivos ${ }^{(7)}$.

Siguiendo lo anterior, el abordaje percutáneo es una opción mínimamente invasiva diferente a la endoscópica, ambas aceptadas por las guías de manejo en pancreatitis aguda ${ }^{(13)}$. En este sentido, el estudio TENSION no encuentra diferencia en las tasas de éxito y las complicaciones al comparar el abordaje mínimamente invasivo endoscópico vs el percutáneo ${ }^{(14)}$. A su vez, en este estudio se evidenció que el 51 \% de los pacientes con abordaje percutáneo no requirió necrosectomía, al igual que el caso clínico presentado.

El abordaje utilizado en el caso descrito fue el drenaje percutáneo transgástrico guiado por tomografía, una técnica de drenaje transvisceral que demuestra ser segura y eficaz ${ }^{(15)}$. Este método posibilita la interacción con el WON mediante lavados con solución salina a través del catéter percutáneo, sin necesidad de realizar nuevos procedimientos que requieran traslado a quirófano e incluso anestesia, asunto frecuente en el abordaje endoscópico. No obstante, una desventaja del abordaje percutáneo podría ser la necesidad de permanencia del catéter determinado según la evolución, siendo necesario su cuidado a través de curaciones diarias, la cuantificación del débito y la no tracción del catéter.

El uso de la ultrasonografía como guía para el abordaje endoscópico o percutáneo está ampliamente descrito para ambas técnicas, siendo segura y efectiva. Esto evitaría la irradiación del paciente, tal como sucede en el caso de la guía tomográfica. Esta técnica tiene la ventaja de posibilitar un control dinámico de los dispositivos durante el procedimiento. En el caso del abordaje percutáneo se necesita inyectar solución salina dentro del estómago para identificar las paredes del estómago y se debe asegurar la vía aérea con necesidad de una anestesia general, para evitar la aspiración del paciente ${ }^{(7,8,16)}$.

El éxito terapéutico puede ser definido de diferentes formas: Nabi et al. ${ }^{(8)}$, lo definen como una reducción mayor del 50 \% del tamaño de la colección y la ausencia de síntomas. De acuerdo con esto, puede pensarse que el abordaje utilizado fue exitoso ya que se disminuyó el tamaño de la colección y los requerimientos de analgesia, además de permitir el inicio inmediato de la alimentación por vía oral. 
El tiempo de permanencia del catéter depende de la evolución clínica y de la cantidad de líquido drenado en 24 horas. Cuando las pérdidas por el catéter se mantienen sin disminuir en cantidad, debe pensarse en una disrupción ductal del páncreas, que ocurre hasta en el $40 \%$ de los pacientes con pancreatitis necrotizante ${ }^{(17,18)}$. En nuestra experiencia el catéter solo se retira si no existe colección residual; sin embargo, en el caso descrito no fue posible definir con exactitud el tiempo de permanencia del catéter, debido a que este fue traccionado involuntariamente y se desplazó al interior de la cavidad gástrica. Una tomografía de control permitió determinar la ausencia de colección, por lo que se retiró el catéter transgástrico.

La posibilidad de recidiva podría llegar al $25 \%{ }^{(19)}$. Aun así, tener una recidiva de una colección peripancreática no es sinónimo de intervención, puesto que, en la mayoría de los casos, el tratamiento puede ser conservador con seguimiento clínico e imagenológico.

\section{Conclusión}

El desarrollo de complicaciones secundarias a la pancreatitis aguda es posible en población pediátrica. El manejo debe realizarse con abordajes mínimamente invasivos según las guías y consensos actuales. El abordaje percutáneo trans-visceral es una técnica segura y eficaz, evitando en algunos casos la necesidad de necrosectomía. Una complicación frecuente es la recidiva de las colecciones, pudiendo ser manejada de forma conservadora en la mayoría de los casos. Aun así, para la evaluación de la efectividad y la morbimortalidad del abordaje transgástrico en la población pediátrica, se requieren estudios multicéntricos y randomizados que posibiliten llegar a una conclusión con mayor evidencia científica.

\section{Financiación}

Ninguna.

\section{Bibliografía}

1. Morinville VD, Barmada MM, Lowe ME. Increasing incidence of acute pancreatitis at an American pediatric tertiary care center: is greater awareness among physicians responsible? Pancreas. 2010 Jan;39(1):5-8. doi: 10.1097/MPA.0b013e3181baac47. PMID: 19752770.

2. Pant C, Deshpande A, Olyaee M, Anderson MP, Bitar A, Steele MI, et al. Epidemiology of acute pancreatitis in hospitalized children in the United States from 2000-2009. PLoS One. 2014;9(5):1-8. 
3. Zaheer Nabi, Rupjyoti Talukdar DNR. Endoscopic management of pancreatic fluid collections. Gut Liver, Vol 11, No 4,. 2017;4(4):474-80.

4. Weizman Z, Durie PR. Acute pancreatitis in childhood. J Pediatr. 1988;113(1 PART 1):249.

5. Bai HX, Lowe ME, Husain SZ. What Have We Learned About Acute Pancreatitis in Children? J Pediatr Gastroenterol Nutr [Internet]. 2011;23(1):1-7. Available from: https://www.ncbi.nlm.nih.gov/pmc/articles/PMC3624763/pdf/nihms412728.pdf

6. Banks PA, Bollen TL, Dervenis C, Gooszen HG, Johnson CD, Sarr MG, et al. Classification of acute pancreatitis - 2012: Revision of the Atlanta classification and definitions by international consensus. Gut. 2013;62(1):102-11.

7. Bang JY, Varadarajulu S. Endoscopic treatment of walled-off necrosis in children: Clinical experience and treatment outcomes. J Pediatr Gastroenterol Nutr. 2016;63(3):e31-5.

8. Nabi Z, Lakhtakia S, Basha J, Chavan R, Ramchandani M, Gupta R, et al. Endoscopic ultrasound-guided drainage of walled-off necrosis in children with fully covered selfexpanding metal stents. J Pediatr Gastroenterol Nutr. 2017;64(4):592-7.

9. Park A, Latif SU, Shah AU, Jianmin T, Werlin S, Hsiao A, et al. Changing Referral Trends of Acute Pancreatitis in Children: A 12-year Single-center Analysis. Pediatr J Gastroenterololy Nutr [Internet]. 2009;23(1):1-7. Available from: https://www.ncbi.nlm.nih.gov/pmc/articles/PMC3624763/pdf/nihms412728.pdf

10. Isaji S, Takada T, Mayumi T, Yoshida M, Wada K, Yokoe M, et al. Revised Japanese guidelines for the management of acute pancreatitis 2015: Revised concepts and updated points. J Hepatobiliary Pancreat Sci. 2015;22(6):433-45.

11. Working group IAP/APA. IAP/APA evidence-based guidelines for the management of acute pancreatitis. Pancreatology. 2013;13(4 SUPPL. 2):1-15.

12. Tenner S, Baillie J, Dewitt J, Vege SS. American College of Gastroenterology guideline: Management of acute pancreatitis. Am J Gastroenterol [Internet]. 2013;108(9):1400-15. Available from: http://dx.doi.org/10.1038/ajg.2013.218

13. Van Santvoort HC, Besselink MG, Bakker OJ, Hofker HS, Boermeester MA, Dejong CH, et al. A step-up approach or open necrosectomy for necrotizing pancreatitis. N Engl J Med. 2010 Apr 22;362(16):1491-502. 
14. Van Brunschot S, van Grinsven J, Voermans RP, Bakker OJ, Besselink MGH, Boermeester $M A$, et al. Transluminal endoscopic step-up approach versus minimally invasive surgical step-up approach in patients with infected necrotising pancreatitis (TENSION trial): Design and rationale of a randomised controlled multicenter trial [ISRCTN09186711]. BMC Gastroenterol. 2013;13(1):1-13.

15. Ramírez J, Arroyave Y, Quilindo C, Romero T, Priarone C. Manejo del drenaje percutáneo guiado por imágenes en un hospital de tercer nivel. Rev Colomb Cirugía. 2019;34(2):16370.

16. Das KM, Kochhar R, Mehta SK, Suri S, Kaushik SP, Gupta NM, et al. Modified ultrasoundguided percutaneous transgastric drainage of pancreatic pseudocysts. Surg Endosc. 1990;4(4):209-11.

17. Varadarajulu S, Wilcox CM. Endoscopic placement of permanent indwelling transmural stents in disconnected pancreatic duct syndrome: Does benefit outweigh the risks? Gastrointest Endosc [Internet]. 2011;74(6):1408-12. Available from: http://dx.doi.org/10.1016/i.gie.2011.07.049

18. Bang JY, Wilcox CM, Navaneethan U, Hasan MK, Peter S, Christein J, et al. Impact of Disconnected Pancreatic Duct Syndrome on the Endoscopic Management of Pancreatic Fluid Collections. Ann Surg. 2018;267(3):561-8.

19. Varadarajulu S, Bang JY, Phadnis MA, Christein JD, Wilcox CM. Endoscopic Transmural Drainage of Peripancreatic Fluid Collections: Outcomes and Predictors of Treatment Success in 211 Consecutive Patients. J Gastrointest Surg. 2011;15(11):2080-8. 